Modern Physics Letters A

(C) World Scientific Publishing Company

\title{
A REVIEW OF INTEGRABLE DEFORMATIONS IN ADS/CFT
}

\author{
IAN SWANSON \\ School of Natural Sciences, Institute for Advanced Study, Einstein Drive \\ Princeton, New Jersey 08540, USA \\ swanson@sns.ias.edu
}

\begin{abstract}
Marginal $\beta$ deformations of $\mathcal{N}=4$ super-Yang-Mills theory are known to correspond to a certain class of deformations of the $S^{5}$ background subspace of type IIB string theory in $A d S_{5} \times S^{5}$. An analogous set of deformations of the $A d S_{5}$ subspace is reviewed here. String energy spectra computed in the near-pp-wave limit of these backgrounds match predictions encoded by discrete, asymptotic Bethe equations, suggesting that the twisted string theory is classically integrable in this regime. These Bethe equations can be derived algorithmically by relying on the existence of Lax representations, and on the Riemann-Hilbert interpretation of the thermodynamic Bethe ansatz. This letter is a review of a seminar given at the Institute for Advanced Study, based on research completed in collaboration with McLoughlin.
\end{abstract}

Electronic version of an article published as [Modern Physics Letters A, Vol. 22, No. 13 (2007) 915-930] [doi:10.1142/S0217732307023614] (C) [copyright World Scientific Publishing Company] [http://www.worldscinet.com/mpla/mpla.shtml].

Keywords: AdS/CFT correspondence; integrable systems.

PACS Nos.: 11.25.Tq, 11.25.Hf, 02.30.Ik

\section{Introduction}

In recent years a large number of studies have emerged indicating that type IIB string theory on $A d S_{5} \times S^{5}$ and $\mathcal{N}=4$ super-Yang-Mills (SYM) theory in four dimensions may be integrable in the planar limit. The technology of integrable systems has therefore become extraordinarily useful in studying the AdS/CFT correspondence in detail. The correspondence equates the spectrum of operator anomalous dimensions of the gauge theory with the energy spectrum of the string theory. The rank of the Yang-Mills gauge group determines the amount of Ramond-Ramond (RR) flux on the $S^{5}$ subspace in the string theory, and in the planar limit this number is scaled to infinity: $N_{c} \rightarrow \infty$. The string coupling $g_{s}$ is related to the gauge theory coupling $g_{\mathrm{YM}}$ via the standard relation, $g_{s}=e^{\phi_{0}}=g_{\mathrm{YM}}^{2} / 4 \pi$, and the radial scale of both the $A d S_{5}$ and $S^{5}$ spaces is given by $R^{4}=4 \pi g_{s} N_{c}=g_{\mathrm{YM}}^{2} N_{c}=\lambda$ (with $\left.\alpha^{\prime}=1\right)$.

If these theories are indeed integrable, the dynamics should be encoded in a diffractionless scattering matrix $S$. On the string side, in the strong-coupling limit $\left(\lambda=g_{\mathrm{YM}}^{2} N_{c} \rightarrow \infty\right)$, this $S$ matrix can be interpreted as describing the two-body 
scattering of elementary excitations on the worldsheet. As their worldsheet momenta becomes large, these excitations are better described as special types of solitonic solutions, or giant magnons, and the interpolating region is described by the dynamics of the so-called near-flat-space regime ${ }^{112}$ On the gauge theory side, the action of the dilatation generator on single-trace operators can be equated with that of a Hamiltonian acting on states of a spin chain! ${ }^{3}$ In this picture, operators in the trace are represented as lattice pseudoparticles that, like their stringy counterparts, experience diffractionless scattering encoded by an $S$ matrix. Proving that the gauge and string theories are identical in the planar limit therefore amounts to showing that the underlying physics of both theories is governed by the same two-body scattering matrix. In fact, symmetry fixes this $S$ matrix up to an overall phase $\sigma$, so what remains is to somehow determine $\sigma$ from basic principles 4 (Unitarity and crossing relations, as they exist in this context, constrain this phase to some extent; see Refs. [5, 6, 7 for recent developments.)

An impressive amount of evidence exists in favor of the mutual integrability of these two theories. If true, this raises the question of whether these theories can be deformed in a controlled manner while remaining integrable. One class of interesting deformations to consider are the marginal $\beta$ deformations of $\mathcal{N}=4$ SYM, also known as Leigh-Strassler deformations. $\frac{9}{}$ The resulting theories comprise a one-parameter family of $\mathcal{N}=1$ conformal gauge theories (in the case of real $\beta$ deformations). On the gravity side of the correspondence, these correspond to special geometrical deformations of the $S^{5}$ subspace in the string theory background 10

In fact, the integrability of the gauge and string theory, to the extent that it is understood in the undeformed cases, seems to persist under these deformations. This problem was studied directly and indirectly, for example, in Refs. 11, 12, 13, 14, 15, 16, 17, 18, 19, 20, 21 (see also references therein). The dynamics of both theories can be captured, at least in certain limits, by twisted Bethe equations. Here we review an analogous class of deformations acting on the $A d S_{5}$ subspace of the string theory background, first studied in Ref. 22. While the corresponding gauge theory is less well understood (it may be a non-commutative or even non-associative theory), the string theory seems to be well defined in the near-pp-wave regime. Furthermore, the string energy spectrum can be computed precisely in this limit from a discrete Bethe ansatz, which lends substantial support to the methodology developed in Refs. 23, 24, 25,

In Section 2 below, TsT deformations of the string background geometry are reviewed in detail. The classical integrability of the string sigma model is discussed in Section 3 . String energy spectra are computed directly from the deformed GreenSchwarz action in the near-pp-wave limit in Section 4. In Section [5 the thermodynamic Bethe equations are promoted to discrete Bethe equations that correctly reproduce the deformed energy spectra. A brief discussion and thoughts on further research are given in Section 6. This letter is a review of a seminar based on Ref. 22 given in May, 2006 at the Institute for Advanced Study. 


\section{Deformations of the string background geometry}

In Ref. 10, it was demonstrated that marginal $\beta$ deformations of $\mathcal{N}=4$ SYM theory correspond to specific deformations of the $A d S_{5} \times S^{5}$ background geometry of the dual gravity theory. In general, these transformations act on global toroidal (or $U(1) \times U(1))$ isometries of the string theory background, and have been dubbed $\gamma$ deformations. The generic action of these deformations can be understood as arising from the following sequence of transformations:

(i) T duality acting on the first $U(1)$ factor of the global symmetry;

(ii) a coordinate shift parameterized by $\gamma$ acting on the second $U(1)$ factor;

(iii) $\mathrm{T}$ duality acting again on the first $U(1)$.

For this reason, these $\gamma$ deformations are also known as TsT deformations.

\subsection{Deformations on the $S^{5}$ subspace}

In keeping with notation used in the literature, we will parameterize the coordinate shift with the symbols $\tilde{\gamma}_{i}$. For the case of real $\tilde{\gamma}_{i}$, the deformed spacetime metric and background fields are given by (mostly following the notation of Ref. 26, and with $\alpha^{\prime}=1$ ),

$$
\begin{aligned}
d s_{\text {string }}^{2} / R^{2} & =d s_{A d S_{5}}^{2}+\sum_{i=1}^{3}\left(d \rho_{i}^{2}+G \rho_{i}^{2} d \phi_{i}^{2}\right)+G \rho_{1}^{2} \rho_{2}^{2} \rho_{3}^{2}\left[d\left(\sum_{i=1}^{3} \tilde{\gamma}_{i} \phi_{i}\right)\right]^{2}, \\
B_{2} & =R^{2} G w_{2}, \quad e^{\phi}=e^{\phi_{0}} G^{1 / 2} \\
w_{2} & \equiv \tilde{\gamma}_{3} \rho_{1}^{2} \rho_{2}^{2} d \phi_{1} \wedge d \phi_{2}+\tilde{\gamma}_{1} \rho_{2}^{2} \rho_{3}^{2} d \phi_{2} \wedge d \phi_{3}+\tilde{\gamma}_{2} \rho_{3}^{2} \rho_{1}^{2} d \phi_{3} \wedge d \phi_{1} \\
G^{-1} & \equiv 1+\tilde{\gamma}_{3}^{2} \rho_{1}^{2} \rho_{2}^{2}+\tilde{\gamma}_{1}^{2} \rho_{2}^{2} \rho_{3}^{2}+\tilde{\gamma}_{2}^{2} \rho_{1}^{2} \rho_{3}^{2} .
\end{aligned}
$$

Here, the $S^{5}$ subspace has undergone three consecutive TsT deformations parameterized by $\tilde{\gamma}_{i}$, with $i \in 1,2,3$. $B_{2}$ is the NS-NS two-form field strength, and the twoand five-form field strengths $C_{2}$ and $F_{5}$ have been omitted. The angular coordinates on the sphere can be written as

$$
\rho_{1}=\sin \alpha \cos \theta, \quad \rho_{2}=\sin \alpha \sin \theta, \quad \rho_{3}=\cos \alpha,
$$

such that $\sum_{i=1}^{3} \rho_{i}^{2}=1$.

We find it convenient to use the following lightcone parametrization on $S^{5}$ :

$$
\begin{aligned}
& \rho_{2}=\frac{y_{1}}{R}, \quad \rho_{3}=\frac{y_{2}}{R}, \quad \rho_{3}=\sqrt{1-\rho_{2}^{2}-\rho_{3}^{2}}, \\
& \phi_{1}=x^{+}+\frac{x^{-}}{R^{2}}, \quad t=x^{+} .
\end{aligned}
$$

At large $R$, we reach a semiclassical limit described by point-like (or "BMN") strings boosted to lightlike momentum $J$ along a geodesic on the deformed $S^{5}$ subspace.27 
The angular momentum $J$ in the $\phi_{1}$ direction is related to the scale radius $R$ by $p_{-} R^{2}=J$, and the lightcone momenta take the form

$$
-p_{+}=\Delta-J, \quad-p_{-}=i \partial_{x^{-}}=\frac{i}{R^{2}} \partial_{\phi_{1}}=-\frac{J}{R^{2}} .
$$

We also find it convenient to work with the following form of the spacetime metric on $A d S_{5}$ :

$$
d s_{A d S_{5}}^{2}=-\left(\frac{1+x^{2} / 4 R^{2}}{1-x^{2} / 4 R^{2}}\right)^{2} d t^{2}+\frac{d x^{2} / R^{2}}{\left(1-x^{2} / 4 R^{2}\right)^{2}},
$$

where $x$ is a vector spanning an $S O(4)$ subspace transverse to the lightcone.

To simplify the projection onto closed $s u(2)$ subsectors of the theory, we introduce the following complex coordinates:

$$
\begin{array}{ll}
y=y_{1} \cos \phi_{2}+i y_{1} \sin \phi_{1}, & \bar{y}=y_{1} \cos \phi_{2}-i y_{1} \sin \phi_{1}, \\
z=y_{2} \cos \phi_{2}+i y_{2} \sin \phi_{1}, & \bar{z}=y_{2} \cos \phi_{2}-i y_{2} \sin \phi_{1} .
\end{array}
$$

Defining the large- $R$ expansion of the spacetime metric by

$$
d s^{2}=d s_{(0)}^{2}+\frac{d s_{(1)}^{2}}{R^{2}}+O\left(1 / R^{4}\right)
$$

we obtain

$$
\begin{aligned}
& d s_{(0)}^{2}=2 d x^{+} d x^{-}+|d y|^{2}+|d z|^{2}-\left(d x^{+}\right)^{2}\left[x^{2}+|y|^{2}\left(1+\tilde{\gamma}_{3}^{2}\right)+|z|^{2}\left(1+\tilde{\gamma}_{2}^{2}\right)\right] \\
& d s_{(1)}^{2}=\left(d x^{-}\right)^{2}+\frac{1}{4}(y d \bar{y}+\bar{y} d y+z d \bar{z}+\bar{z} d z)^{2}+\frac{1}{2} x^{2} d x^{2} \\
& \quad+\left(d x^{+}\right)^{2}\left[-\frac{1}{2} x^{4}+2\left(|z|^{2}+|y|^{2}\right)\left(|z|^{2} \tilde{\gamma}_{2}^{2}+|y|^{2} \tilde{\gamma}_{3}^{2}\right)+\left(|y|^{2} \tilde{\gamma}_{3}^{2}+|z|^{2} \tilde{\gamma}_{2}^{2}\right)^{2}\right] \\
& \quad+\tilde{\gamma}_{1} d x^{+}\left(\tilde{\gamma}_{2}|z|^{2} \operatorname{Im}(\bar{y} d y)+\tilde{\gamma}_{3}|y|^{2} \operatorname{Im}(\bar{z} d z)\right)-\tilde{\gamma}_{3}^{2} \operatorname{Im}(\bar{y} d y)^{2}-\tilde{\gamma}_{3}^{2} \operatorname{Im}(\bar{z} d z)^{2} \\
& \quad+\tilde{\gamma}_{2} \tilde{\gamma}_{3} \operatorname{Im}(\bar{y} d y) \operatorname{Im}(\bar{z} d z)-2 d x^{+} d x^{-}\left(|y|^{2}\left(1+\tilde{\gamma}_{3}^{2}\right)+|z|^{2}\left(1+\tilde{\gamma}_{2}^{2}\right)\right) .
\end{aligned}
$$

As expected, the pp-wave metric appears at leading order in the expansion. The corresponding expansion of the NS-NS two-form $B_{2}$ appears as

$$
\begin{aligned}
& B_{2}=\tilde{\gamma}_{3} d x^{+} \wedge \operatorname{Im}(\bar{y} d y)-\tilde{\gamma}_{2} d x^{+} \wedge \operatorname{Im}(\bar{z} d z) \\
& \quad+\frac{1}{R^{2}}\left[-\tilde{\gamma}_{3}\left(\tilde{\gamma}_{3}^{2}|y|^{2}+\tilde{\gamma}_{2}^{2}|z|^{2}\right) d x^{+} \wedge \operatorname{Im}(\bar{y} d y)+\tilde{\gamma}_{2}\left(\tilde{\gamma}_{3}^{2}|y|^{2}+\tilde{\gamma}_{2}^{2}|z|^{2}\right) d x^{+} \wedge \operatorname{Im}(\bar{z} d z)\right. \\
& \left.\quad+\tilde{\gamma}_{3} d x^{-} \operatorname{Im}(\bar{y} d y)-\tilde{\gamma}_{2} d x^{-} \wedge \operatorname{Im}(\bar{z} d z)+\tilde{\gamma}_{1} \operatorname{Im}(\bar{y} d y) \wedge \operatorname{Im}(\bar{z} d z)\right] .
\end{aligned}
$$

The rank-one $s u(2)$ subsector decouples from the theory in the near-pp-wave limit, though it has nontrivial dynamics itself. We can therefore study is as a separate theory, without having to worry about mixing. The TsT-deformed version, labeled by $s u(2)_{\gamma}$, can be reached by projecting onto a single complex coordinate (either $y$ or $z$ for the parametrization given above):

$$
\begin{aligned}
& d s_{s u(2)_{\gamma}}^{2}=2 d x^{+} d x^{-}-\left(1+\tilde{\gamma}^{2}\right)|y|^{2}\left(d x^{+}\right)^{2}+|d y|^{2}+\frac{1}{R^{2}}\left[\frac{1}{4}(y d \bar{y}+\bar{y} d y)^{2}\right. \\
& \left.+\left(d x^{-}\right)^{2}+\tilde{\gamma}^{2}\left(2+\tilde{\gamma}^{2}\right)|y|^{4}\left(d x^{+}\right)^{2}-2\left(1+\tilde{\gamma}^{2}\right)|y|^{2} d x^{+} d x^{-}-\tilde{\gamma} \operatorname{Im}(\bar{y} d y)\right],
\end{aligned}
$$


where we have truncated the series at $O\left(1 / R^{4}\right)$. Here we allow the parameter $\tilde{\gamma}$ to stand for either $\tilde{\gamma}_{2}$ or $\tilde{\gamma}_{3}$, corresponding to two possible choices of $s u(2)_{\gamma}$ truncation. The corresponding NS-NS two-form reduces to

$$
\begin{aligned}
B_{2}^{s u(2)_{\gamma}}= & \tilde{\gamma} d x^{+} \wedge \operatorname{Im}(\bar{y} d y)+\frac{\tilde{\gamma}}{R^{2}}\left(d x^{-} \wedge \operatorname{Im}(\bar{y} d y)\right. \\
& \left.-\left(1+\tilde{\gamma}^{2}\right)|y|^{2} d x^{+} \wedge \operatorname{Im}(\bar{y} d y)\right)+O\left(1 / R^{4}\right) .
\end{aligned}
$$

\subsection{Deformations in the $A d S_{5}$ subspace}

To study analogous deformations of the $A d S_{5}$ subspace, it is convenient to start with an $S O(4,2)$ invariant, expressed in terms of $\mathbb{R}^{6}$ embedding coordinates:

$$
-X_{0}^{2}+X_{1}^{2}+X_{2}^{2}+X_{3}^{2}+X_{4}^{2}-X_{5}^{2}=-1
$$

such that

$$
\begin{array}{ll}
X_{0}=\eta_{1} \sin \hat{\varphi}_{1}, & X_{1}=\eta_{2} \cos \hat{\varphi}_{2}, \\
X_{2}=\eta_{2} \sin \hat{\varphi}_{2}, & X_{3}=\eta_{3} \cos \hat{\varphi}_{3}, \\
X_{4}=\eta_{3} \sin \hat{\varphi}_{3}, & X_{5}=\eta_{1} \cos \hat{\varphi}_{1} .
\end{array}
$$

The $\hat{\varphi}_{i}$ variables denote untwisted $U(1)$ angular coordinates. One can make contact with the more familiar angular coordinates on $A d S_{5}$ using

$$
\eta_{1}=\cosh \alpha, \quad \eta_{2}=\sinh \alpha \sin \theta, \quad \eta_{3}=\sinh \alpha \cos \theta .
$$

This preserves the $S O(2,1)$ invariant $-\eta_{1}^{2}+\eta_{2}^{2}+\eta_{3}^{2}=-1$. From the resulting metric

$$
\begin{aligned}
d s_{A d S_{5}}^{2} / R^{2} & =-\left(d \eta_{1}^{2}+\eta_{1}^{2} d \hat{\varphi}_{1}^{2}\right)+\sum_{i=2}^{3}\left(d \eta_{i}^{2}+\eta_{i}^{2} d \hat{\varphi}_{i}^{2}\right) \\
& =d \alpha^{2}-\cosh \alpha^{2} d \hat{\varphi}_{1}^{2}+\sinh \alpha^{2}\left(d \theta^{2}+\sin \theta^{2} d \hat{\varphi}_{2}^{2}+\cos \theta^{2} d \hat{\varphi}_{3}^{2}\right),
\end{aligned}
$$

it is easy to see that there is a $U(1) \times U(1) \times U(1)$ global symmetry. TsT deformations may thus act on the corresponding angular coordinates $\hat{\varphi}_{i}(i \in 1,2,3)$. It turns out that we can avoid the usual difficulties associated with T-duality along compact timelike directions (see Ref. 22 for details). In the end, however, one must pass to the universal covering space of the geometry, where the timelike directions are noncompact.

For present purposes, we wish to study a TsT transformation that acts as a Tduality along the $\hat{\varphi}_{2}$ direction, followed by a shift in the $\hat{\varphi}_{1}$ direction $\hat{\varphi}_{1} \rightarrow \hat{\varphi}_{1}+\tilde{\gamma} \hat{\varphi}_{2}$, and a final T-duality along the new $\varphi_{2}$ direction (where the notation $\varphi_{2}$ indicates a transformed angular coordinate). The deformed metric thus takes the form

$$
d s_{\mathrm{str}}^{2} / R^{2}=d s_{S^{5}}^{2}+g^{i j} d \eta_{i} d \eta_{j}+g^{i j} G \eta_{i}^{2} d \varphi_{j}^{2}-\tilde{\gamma}^{2} G \eta_{1}^{2} \eta_{2}^{2} \eta_{3}^{2} d \varphi_{1}^{2},
$$

with $g=\operatorname{diag}(-1,1,1)$. The NS-NS two-form is

$$
B_{2}=\tilde{\gamma} G \eta_{1}^{2} \eta_{2}^{2} d \varphi_{1} \wedge d \varphi_{2}
$$


where the deformation factor $G$ is given by

$$
G^{-1} \equiv 1-\tilde{\gamma}^{2} \eta_{1}^{2} \eta_{2}^{2}
$$

We focus on the local region for which $\varphi_{2}$ is spacelike: in the end this yields a worldsheet Hamiltonian that appears to be completely consistent.

The $A d S_{5}^{\tilde{\gamma}}$ worldsheet action appears as

$$
\begin{aligned}
& S_{A d S_{5} \tilde{\gamma}}=-\frac{\sqrt{\lambda}}{2} \int d \tau \frac{d \sigma}{2 \pi}\left[\gamma ^ { \alpha \beta } \left(g^{i j} \partial_{\alpha} \eta_{i} \partial_{\beta} \eta_{j}+g^{i j} G \eta_{i}^{2} \partial_{\alpha} \varphi_{j} \partial_{\beta} \varphi_{j}\right.\right. \\
& \left.\left.-\tilde{\gamma}^{2} G \eta_{1}^{2} \eta_{2}^{2} \eta_{3}^{2} \partial_{\alpha} \varphi_{1} \partial_{\beta} \varphi_{1}\right)-2 \epsilon^{\alpha \beta}\left(\tilde{\gamma} G \eta_{1}^{2} \eta_{2}^{2} \partial_{\alpha} \varphi_{1} \partial_{\beta} \varphi_{2}+\Lambda\left(g^{i j} \eta_{i} \eta_{j}+1\right)\right)\right],
\end{aligned}
$$

where $\Lambda$ acts as a Lagrange multiplier enforcing $-\eta_{1}^{2}+\eta_{2}^{2}+\eta_{3}^{2}=-1$ on shell. The indices $\alpha$ and $\beta$ run over the $\tau(\alpha, \beta=0)$ and $\sigma(\alpha, \beta=1)$ directions on the worldsheet, and $\gamma^{\alpha \beta}$ is the worldsheet metric.

To simplify the projection onto closed $s l(2)_{\gamma}$ subsectors (i.e., the analogues of the closed $s u(2)_{\gamma}$ subsectors in the $S^{5}$ case), we choose the lightcone parametrization

$$
\begin{aligned}
\eta_{2} & =\frac{u_{1}}{R}, \quad \eta_{3}=\frac{u_{2}}{R}, \\
\eta_{3} & =\sqrt{1+\eta_{2}^{2}+\eta_{3}^{2}}, \quad \varphi_{1}=x^{+}+\frac{x^{-}}{R^{2}}, \quad t=x^{+},
\end{aligned}
$$

and introduce a new set of complex coordinates:

$$
\begin{aligned}
v & =u_{1} \cos \varphi_{2}+i u_{1} \sin \varphi_{1}, & & \bar{v}=u_{1} \cos \varphi_{2}-i u_{1} \sin \varphi_{1}, \\
w & =u_{2} \cos \varphi_{2}+i u_{2} \sin \varphi_{1}, & & \bar{w}=u_{2} \cos \varphi_{2}-i u_{2} \sin \varphi_{1}
\end{aligned}
$$

Under one such projection, the metric and NS-NS two-form become:

$$
\begin{aligned}
d s_{s l(2)_{\gamma}}^{2}= & 2 d x^{+} d x^{-}-\left(1+\tilde{\gamma}^{2}\right)|v|^{2}\left(d x^{+}\right)^{2}+|d v|^{2}-\frac{1}{R^{2}}\left[\frac{1}{4}(v d \bar{v}+\bar{v} d v)^{2}-\left(d x^{-}\right)^{2}\right. \\
& \left.+\tilde{\gamma}^{2}\left(2+\tilde{\gamma}^{2}\right)|v|^{4}\left(d x^{+}\right)^{2}+\tilde{\gamma}^{2}(v d \bar{v}-\bar{v} d v)^{2}\right]+O\left(1 / R^{4}\right), \\
B_{2}^{s l(2)_{\gamma}}= & \frac{i}{2} \tilde{\gamma} d x^{+} \wedge(v d \bar{v}-\bar{v} d v)+\frac{i}{2 R^{2}}|v|^{2} \tilde{\gamma}\left(1+\tilde{\gamma}^{2}\right) d x^{+} \wedge(\bar{v} d v-v d \bar{v})+O\left(1 / R^{4}\right) .
\end{aligned}
$$

The conserved $U(1)$ currents $J_{i}^{\alpha}$ in the undeformed theory turn out to be identical to those in the deformed theory. By defining canonical momenta as $p_{i}=J_{i}^{0}$, the associated charges take the form $J_{i}=\int \frac{d \sigma}{2 \pi} p_{i}$. The identification $\hat{J}_{i}=J_{i}$ therefore yields

$$
\hat{\varphi}_{1}^{\prime}=\varphi_{1}^{\prime}-\gamma p_{2}, \quad \hat{\varphi}_{2}^{\prime}=\varphi_{2}^{\prime}+\gamma p_{1}, \quad \hat{\varphi}_{3}^{\prime}=\varphi_{3}^{\prime} .
$$

where $\varphi^{\prime}$ denotes a worldsheet $\sigma$ derivative acting on $\varphi$, and we have introduced the rescaled deformation parameter $\gamma \equiv \frac{\tilde{\gamma}}{\sqrt{\lambda}}$. We therefore obtain the following set of twisted boundary conditions

$$
\begin{aligned}
& \hat{\varphi}_{1}(2 \pi)-\hat{\varphi}_{1}(0)=2 \pi\left(m_{1}-\gamma J_{2}\right), \\
& \hat{\varphi}_{2}(2 \pi)-\hat{\varphi}_{2}(0)=2 \pi\left(m_{2}+\gamma J_{1}\right), \\
& \hat{\varphi}_{3}(2 \pi)-\hat{\varphi}_{3}(0)=2 \pi m_{3},
\end{aligned}
$$

where the $m_{i}$ are winding numbers, satisfying $2 \pi m_{i}=\varphi_{i}(2 \pi)-\varphi_{i}(0)$. 


\section{Classical integrability}

We will now review how the classical integrability of the theory on the $A d S_{5}$ subspace is preserved under the deformation described above, relying on a parametrization of the bosonic coset space $(S O(4,2) \times S O(6)) /(S O(5,1) \times S O(5))$ originally given in Ref. 28. The $A d S_{5}$ sector takes the form

$$
g=\left(\begin{array}{cccc}
0 & Z_{1} & -Z_{3} & \bar{Z}_{2} \\
-Z_{1} & 0 & Z_{2} & \bar{Z}_{3} \\
Z_{3} & -Z_{2} & 0 & -\bar{Z}_{1} \\
-\bar{Z}_{2} & -\bar{Z}_{3} & \bar{Z}_{1} & 0
\end{array}\right), \quad Z_{i} \equiv \eta_{i} e^{i \hat{\varphi}_{i}}
$$

which satisfies

$$
g^{\dagger} s g=s, \quad s \equiv \operatorname{diag}(-1,-1,1,1) .
$$

The result is that $g$ is an $S U(2,2)$ embedding of an element of the coset $S O(4,2) / S O(5,1)$. We can therefore work from the principal chiral model defined by

$$
S=\int d \tau d \sigma \gamma^{\alpha \beta} \operatorname{Tr}\left(g^{-1} \partial_{\alpha} g g^{-1} \partial_{\beta} g\right)
$$

Key to the analysis is the existence of a Lax representation, which encodes the equations of motion $\partial_{\alpha}\left(\gamma^{\alpha \beta} j_{\beta}\right)=0$ in an auxiliary linear problem, subject to a constraint equation in the form of a commutator of Lax operators $\left[D_{\alpha}, D_{\beta}\right]=0$. In the case at hand, the Lax operator $D_{\alpha}$ can be defined in terms of a spectral parameter $x$ by

$$
D_{\alpha}=\partial_{\alpha}-\frac{j_{\alpha}^{+}}{2(x-1)}+\frac{j_{\alpha}^{-}}{2(x+1)} \equiv \partial_{\alpha}-\mathcal{A}_{\alpha}(x) .
$$

$\mathcal{A}_{\alpha}(x)$ is the right Lax connection, and the $j_{\alpha}^{+}$and $j_{\alpha}^{-}$are respectively self-dual and anti-self-dual projections of the right current $j_{\alpha}=g^{-1} \partial_{\alpha} g$. It turns out that the non-derivative dependence of the Lax current $j_{\alpha}$ on the angular coordinates $\hat{\varphi}_{i}$ can be gauged away using an invertible matrix $M$

$$
\begin{aligned}
\tilde{\jmath}_{\alpha}\left(\eta_{i}, \partial \hat{\varphi}_{i}\right) & =M j_{\alpha}\left(\eta_{i}, \hat{\varphi}_{i}\right) M^{-1} \\
& =\tilde{g}^{-1} \partial_{\alpha} \tilde{g}+\tilde{g}^{-1} \partial_{\alpha} \Phi \tilde{g}+\partial_{\alpha} \Phi .
\end{aligned}
$$

We now truncate to the deformed $s l(2)_{\gamma}$ sector of the theory. Strings in this subsector propagate on $A d S_{3} \times S^{1}$, though the $S^{1}$ factor will decouple. A useful coordinate parametrization can be found using the following $S L(2)$ matrix:

$$
g=\left(\begin{array}{c}
\cos \hat{\varphi}_{1} \cosh \rho+\cos \hat{\varphi}_{2} \sinh \rho \sin \hat{\varphi}_{1} \cosh \rho-\sin \hat{\varphi}_{2} \sinh \rho \\
-\sin \hat{\varphi}_{1} \cosh \rho-\sin \hat{\varphi}_{2} \sinh \rho \cos \hat{\varphi}_{1} \cosh \rho-\cos \hat{\varphi}_{2} \sinh \rho
\end{array}\right) .
$$

In this case we can invoke a gauge transformation of the form

$$
g=e^{\frac{i}{2}\left(\hat{\varphi}_{1}+\hat{\varphi}_{2}\right) \sigma_{2}} e^{\rho \sigma_{3}} e^{\frac{i}{2}\left(\hat{\varphi}_{1}-\hat{\varphi}_{2}\right) \sigma_{2}},
$$


which eliminates any linear dependence on the coordinates $\hat{\varphi}_{i}\left(\sigma_{i}\right.$ are the usual Pauli matrices). With $M=e^{\frac{i}{2}\left(\hat{\varphi}_{1}-\hat{\varphi}_{2}\right) \sigma_{3}}$, the right current takes the form

$$
\begin{aligned}
\tilde{J}_{\alpha}\left(\eta_{i}, \partial \hat{\varphi}_{i}\right) & =M j_{\alpha}\left(\eta_{i}, \hat{\varphi}_{i}\right) M^{-1} \\
= & \left(\begin{array}{cc}
\partial_{\alpha} \rho & e^{-\rho}\left(\partial_{\alpha} \hat{\varphi}_{1} \cosh \rho-\partial_{\alpha} \hat{\varphi}_{2} \sinh \rho\right) \\
-e^{-\rho}\left(\partial_{\alpha} \hat{\varphi}_{1} \cosh \rho+\partial_{\alpha} \hat{\varphi}_{2} \sinh \rho\right) & -\partial_{\alpha} \rho
\end{array}\right) .
\end{aligned}
$$

We thus find the following local Lax operator and associated Lax connection:

$$
\begin{aligned}
& D_{\alpha} \rightarrow M D_{\alpha} M^{-1} \equiv \partial_{\alpha}-\mathcal{R}_{\alpha}, \\
& \mathcal{R}_{\alpha}=M \mathcal{A}_{\alpha} M^{-1}-M \partial_{\alpha} M^{-1}=\tilde{\mathcal{A}}_{\alpha}+\frac{i}{2}\left(\partial_{\alpha} \hat{\varphi}_{1}-\partial_{\alpha} \hat{\varphi}_{2}\right) \sigma_{2} .
\end{aligned}
$$

Thermodynamic Bethe equations can be derived to encode the spectral problem by studying the pole structure and the asymptotics of the quasimomentum $p(x)$ on the complex spectral $x$-plane. The quasimomentum $p(x)$ is defined in the usual fashion, in terms of a monodromy $\Omega(x)$, according to

$$
\operatorname{Tr} \Omega(x)=2 \cos p(x),
$$

where

$$
\Omega(x)=\mathcal{P} \exp \int_{0}^{2 \pi} d \sigma \mathcal{R}_{1}(x) .
$$

The general strategy is to reformulate the Bethe ansatz as a Riemann-Hilbert problem.29 The gauge freedom noted above turns out to be advantageous when applying these techniques in the presence of $\gamma$ deformations. In fact, we find that the poles of the quasimomentum at $x= \pm 1$ are invariant under $\gamma$ deformations:

$$
p(x)=\pi \frac{J / \sqrt{\lambda} \mp m}{x \pm 1}+\cdots \quad x \rightarrow \mp 1,
$$

where $m$ indicates a winding number associated with the decoupled $S^{1}$ in the $A d S_{3} \times$ $S^{1}$ subspace.

Following the treatment in Ref. 11, we find that it is easiest to study the asymptotics of the problem by using an inverse gauge transformation and relying on the original Lax connection $\mathcal{A}_{\alpha}$ :

$$
T(x)=M(2 \pi) \mathcal{P} \exp \int_{0}^{2 \pi} d \sigma \mathcal{A}_{1}(x) M^{-1}(0) .
$$

This gives a representation of $p(x)$ of the form

$$
\begin{aligned}
2 \cos p(x) & =\operatorname{Tr} M_{R} \mathcal{P} \exp \int_{0}^{2 \pi} d \sigma \mathcal{A}_{1}(x), \\
\mathcal{A}_{1}(x) & =\frac{j_{1}}{x^{2}-1}+\frac{x j_{0}}{x^{2}-1}
\end{aligned}
$$

where

$$
M_{R}=M^{-1}(0) M(2 \pi)=\left(\begin{array}{cc}
\cos \gamma \pi(S-\Delta) & -\sin \gamma \pi(S-\Delta) \\
\sin \gamma \pi(S-\Delta) & \cos \gamma \pi(S-\Delta)
\end{array}\right)
$$


Here we have defined $J_{1}=-\Delta$ and $J_{2}=S$ to make contact with the energy $\Delta$ and impurity number $S$.

Using the right and left currents

$$
j_{\alpha}=g^{-1} \partial_{\alpha} g=\frac{1}{2} j_{\alpha} \cdot \hat{\sigma}, \quad l_{\alpha}=\partial_{\alpha} g g^{-1}=\frac{1}{2} l_{\alpha} \cdot \hat{\sigma},
$$

with $\hat{\sigma} \equiv\left(i \sigma_{2}, \sigma_{3},-\sigma_{1}\right)$, we find

$$
\frac{\sqrt{\lambda}}{4 \pi} \int_{0}^{2 \pi} d \sigma j_{0}^{0}=\Delta+S, \quad \frac{\sqrt{\lambda}}{4 \pi} \int_{0}^{2 \pi} d \sigma l_{0}^{0}=\Delta-S .
$$

At this point we may simply expand $p(x)$ in the asymptotic regions and, following the prescription described in Ref. 11, discard nonlocal contributions to recover

$$
\begin{array}{ll}
p(x)=\pi \gamma(\Delta-S)+2 \pi \frac{\Delta+S}{\sqrt{\lambda} x}+\cdots, & x \rightarrow \infty, \\
p(x)=\pi \gamma(\Delta+S)-2 \pi \frac{\Delta-S}{\sqrt{\lambda}} x+\cdots, & x \rightarrow 0 .
\end{array}
$$

With the above input, one may now define a resolvent:

$$
G(x)=p(x)-\pi \frac{J / \sqrt{\lambda}+m}{x-1}-\pi \frac{J / \sqrt{\lambda}-m}{x+1}-\pi \gamma(\Delta-S) .
$$

The asymptotics of $G(x)$ are completely determined by the corresponding behavior of $p(x)$. We find

$$
\begin{aligned}
& G(x)=\frac{2 \pi}{\sqrt{\lambda} x}(\Delta+S-J)+\cdots, \quad x \rightarrow \infty \\
& G(x)=2 \pi(m+\gamma S)+\frac{2 \pi x}{\sqrt{\lambda}}(S-\Delta+J)+\cdots, \quad x \rightarrow 0 .
\end{aligned}
$$

The next step is to compare this with the usual spectral representation

$$
G(x)=\int_{C} d x^{\prime} \frac{\sigma\left(x^{\prime}\right)}{x-x^{\prime}}, \quad C=C_{1} \cup C_{2} \ldots \cup C_{N}, \quad x \in C_{k},
$$

where $\sigma(x)$ is a spectral density function supported on a finite number of cuts in the $x$ plane denoted by $C_{k}$. Using the analyticity of $G$, we derive the following constraints

$$
\begin{aligned}
& \int_{C} d x \sigma(x)=\frac{2 \pi}{\sqrt{\lambda}}(\Delta+S-J), \\
& \int_{C} d x \frac{\sigma(x)}{x}=-2 \pi(m+\gamma S), \\
& \int_{C} d x \frac{\sigma(x)}{x^{2}}=\frac{2 \pi}{\sqrt{\lambda}}(\Delta-S-J) .
\end{aligned}
$$

These may be combined with the condition

$$
p(x+i 0)+p(x-i 0)=2 \pi n_{k}, \quad x \in C_{k},
$$


which can be understood as arising from the unimodularity of $\Omega(x)$. The mode numbers $n_{k}$ denote eigenvalues that are supported on the $k^{\text {th }}$ contour.

The conditions above yield the following finite-gap integral equation:

$$
\begin{aligned}
& 2 \pi\left(n_{k}-\gamma J\right)-4 \pi \frac{x J / \sqrt{\lambda}}{x^{2}-1}= \\
& \quad 2 f_{C} d x^{\prime} \sigma\left(x^{\prime}\right)\left(\frac{1}{x-x^{\prime}}-\frac{2 x^{\prime}+\gamma \sqrt{\lambda}\left(x^{\prime 2}-1\right)}{2 x^{\prime 2}\left(x^{2}-1\right)}+\frac{\gamma \sqrt{\lambda}}{2} \frac{1}{x^{\prime 2}}\right) .
\end{aligned}
$$

Relative to the undeformed theory in the limit $\gamma \rightarrow 0$, we have acquired an overall shift in the mode number $n_{k}$ proportional to $\gamma$, as well as a number of $\gamma$-dependent modifications appearing in the integrand. In the following sections we will demonstrate that this thermodynamic Bethe equation can be promoted to a discrete Bethe equation that reliably encodes string energy spectra in the near-pp-wave limit. To do this, we must first gather spectral data directly from the string theory.

\section{String spectra}

The canonical, gauge-fixed lightcone Hamiltonian in the bosonic $s u(2)_{\gamma}$ sector of the string theory in the near-pp-wave limit can be split into a free quadratic theory (the full pp-wave limit) and an interaction correction, according to

$$
H_{\mathrm{LC}}=H_{0}+\frac{H_{\mathrm{int}}}{R^{2}}+O\left(1 / R^{4}\right) .
$$

Following the methods described in detail in Refs. 30, 31, 32, 33, we find the following explicit expressions in terms of worldsheet fields:

$$
\begin{aligned}
H_{0}\left(S_{\tilde{\gamma}}^{5}\right)= & \frac{1}{2 p_{-}}\left[4\left|p_{y}\right|^{2}+\left|y^{\prime}\right|^{2}-i p_{-}\left(y^{\prime} \bar{y}-y \bar{y}^{\prime}\right) \tilde{\gamma}+p_{-}^{2}|y|^{2}\left(1+\tilde{\gamma}^{2}\right)\right], \\
H_{\text {int }}\left(S_{\tilde{\gamma}}^{5}\right)= & \frac{1}{8 p_{-}^{3}}\left\{-4 p_{y}^{2}\left(4 \bar{p}_{y}^{2}+p_{-}^{2} y^{2}-y^{\prime 2}\right)-16 p_{-}^{2}\left|p_{y}\right|^{2}|y|^{2}\right. \\
& +p_{-}^{2} \bar{y}^{2}\left(3 p_{-}^{2} y^{2}+y^{\prime 2}-4 \bar{p}_{y}^{2}\right)+\bar{y}^{\prime 2}\left(p_{-}^{2} y^{2}-y^{\prime 2}+4 \bar{p}_{y}^{2}\right) \\
& -2 i p_{-} \tilde{\gamma}\left[-4 p_{y}^{2} y y^{\prime}+p_{-}^{2}|y|^{2}\left(y \bar{y}^{\prime}-\bar{y} y^{\prime}\right)+\bar{y}^{\prime}\left(4 \bar{p}_{y}^{2} \bar{y}-y^{\prime 2} \bar{y}+y\left|y^{\prime}\right|^{2}\right)\right] \\
& -p_{-}^{2} \tilde{\gamma}^{2}\left[4 p_{y}^{2} y^{2}+\bar{y}^{2}\left(4 \bar{p}_{y}^{2}+2 p_{-}^{2} y^{2}-y^{\prime 2}\right)+4|y|^{2}\left|y^{\prime}\right|^{2}-y^{2} \bar{y}^{\prime 2}\right. \\
& \left.\left.+2 i p_{-} \tilde{\gamma}|y|^{2}\left(y \bar{y}^{\prime}-y^{\prime} \bar{y}\right)+p_{-}^{2} \tilde{\gamma}^{2}|y|^{4}\right]\right\} .
\end{aligned}
$$

In truncating to the $s u(2)_{\gamma}$ sector, we have projected onto the complex $y$ coordinates (though this does not achieve the complete projection). The free Hamiltonian can be solved and quantized exactly, yielding the following dispersion relations:

$$
\omega_{n}^{2}=p_{-}^{2}+\left(n-p_{-} \tilde{\gamma}\right)^{2}, \quad \bar{\omega}_{n}^{2}=p_{-}^{2}+\left(n+p_{-} \tilde{\gamma}\right)^{2},
$$

where the integers $n$ are mode indices. To complete the projection to the closed $s u(2)_{\gamma}$ subsector, we keep one set of bosonic raising and lowering Fourier modes, 
either $\left(a_{n}, a_{-n}^{\dagger}\right)$ or $\left(\bar{a}_{n}, \bar{a}_{-n}^{\dagger}\right)$. We thus choose a basis of unperturbed string energy eigenstates spanned by

$$
a_{n_{1}}^{\dagger} a_{n_{1}}^{\dagger} \cdots a_{n_{N}}^{\dagger}|J\rangle
$$

where $|J\rangle$ is understood to be a ground state carrying angular momentum $J$ on the $S^{5}$.

These states carry conserved impurity number $N$, labeled by $N$ integer mode numbers $n_{j}$. We choose to organize these numbers such that the set $\left\{n_{j}\right\}$ contains $M$ subsets of $N_{j}$ equivalent mode numbers $n_{j}$, with $j \in 1, \ldots, M$ :

$$
\left\{n_{j}\right\}=\left\{\{\underbrace{n_{1}, n_{1}, \ldots, n_{1}}_{N_{1}}\},\{\underbrace{n_{2}, n_{2}, \ldots, n_{2}}_{N_{2}}\}, \ldots,\{\underbrace{n_{M}, n_{M}, \ldots, n_{M}}_{N_{M}}\}\right\} .
$$

With the identifications $J=p_{-} R^{2}$ and $p_{-}=1 / \sqrt{\lambda^{\prime}}$, which hold in the near-ppwave limit, we arrange the large- $J$ expansion of energy eigenvalues according to the formula

$$
E\left(\left\{n_{j}\right\}, J\right)=\sum_{j=1}^{N} \sqrt{1+\left(n_{j}-\tilde{\gamma} / \sqrt{\lambda^{\prime}}\right)^{2} \lambda^{\prime}}+\delta E\left(\left\{n_{j}\right\}, J\right)+O\left(1 / J^{2}\right) .
$$

Upon diagonalizing the Hamiltonian, we find the following interaction correction to the free theory in the $s u(2)_{\gamma}$ sector:

$$
\begin{gathered}
\delta E_{s u(2)_{\gamma}}\left(\left\{n_{j}\right\},\left\{N_{j}\right\}, J\right)=-\frac{1}{2 J}\left\{\sum_{j=1}^{M} N_{j}\left(N_{j}-1\right)\left[\left(1+\left(\tilde{\gamma}-n_{j} \sqrt{\lambda^{\prime}}\right)^{-2}\right)^{-1}\right]\right. \\
-\sum_{\substack{j, k=1 \\
j \neq k}}^{M} \frac{N_{j} N_{k}}{\omega_{n_{j}} \omega_{n_{k}} \lambda^{\prime}}\left\{-\lambda^{\prime}\left(n_{j} n_{k}+n_{k}^{2}+n_{j}^{2}\left(1+n_{k}^{2} \lambda^{\prime}\right)\right)\right. \\
+\tilde{\gamma}\left(\left(n_{j}+n_{k}\right) \sqrt{\lambda^{\prime}}-\tilde{\gamma}\right)\left(3+2 n_{j} n_{k} \lambda^{\prime}-\left(n_{j}+n_{k}\right) \sqrt{\lambda^{\prime}} \tilde{\gamma}+\tilde{\gamma}^{2}\right) \\
\left.\left.+\lambda^{\prime}\left(n_{j} \sqrt{\lambda^{\prime}}-\tilde{\gamma}\right)\left(n_{k} \sqrt{\lambda^{\prime}}-\tilde{\gamma}\right) \omega_{n_{j}} \omega_{n_{k}}\right\}\right\} .
\end{gathered}
$$

In fact, this expression is identical to the one first computed in Ref. 34, with a global shift in the mode numbers $n_{j} \rightarrow n_{j}-\beta J$, where $\beta=\gamma=\tilde{\gamma} / \sqrt{\lambda}$.

In the deformed $s l(2)_{\gamma}$ sector we find a near-pp-wave canonical Hamiltonian similar to the $s u(2)_{\gamma}$ case described above:

$$
\begin{aligned}
& H_{0}\left(A d S_{5}^{\tilde{\gamma}}\right)=\frac{1}{2 p_{-}}\left[4\left|p_{v}\right|^{2}+\left|v^{\prime}\right|^{2}-i p_{-}\left(v^{\prime} \bar{v}-v \bar{v}^{\prime}\right) \tilde{\gamma}+p_{-}^{2}|v|^{2}\left(1+\tilde{\gamma}^{2}\right)\right], \\
& H_{\mathrm{int}}\left(A d S_{5}^{\tilde{\gamma}}\right)=\frac{1}{8 p_{-}^{3}}\left\{16 p_{-}^{2}\left|p_{v}\right|^{2}|v|^{2}+\left(4 \bar{p}_{v}^{2}-v^{\prime 2}\right) \bar{v}^{\prime 2}+4 i p_{-}^{3}|v|^{2}\left(v \bar{v}^{\prime}-v^{\prime} \bar{v}\right) \tilde{\gamma}\left(1+\tilde{\gamma}^{2}\right)\right. \\
& \quad+p_{-}^{4}|v|^{4}\left(-1+6 \tilde{\gamma}^{2}+3 \tilde{\gamma}^{4}\right)+4 p_{v}^{2}\left(-4 \bar{p}_{v}^{2}+v^{\prime 2}+p_{-}^{2} v^{2}\left(1+\tilde{\gamma}^{2}\right)\right) \\
& \left.\quad+p_{-}^{2}\left[4|v|^{2}\left|v^{\prime}\right|^{2} \tilde{\gamma}^{2}+4 \bar{p}_{v}^{2} \bar{v}^{2}\left(1+\tilde{\gamma}^{2}\right)-v^{\prime 2} \bar{v}^{2}\left(1+\tilde{\gamma}^{2}\right)-v^{2} \bar{v}^{\prime 2}\left(1+\tilde{\gamma}^{2}\right)\right]\right\} .
\end{aligned}
$$


By again solving the free limit of the theory, expanding in Fourier modes and projecting completely onto the closed $s l(2)_{\gamma}$ sector, we find the following correction to the energy spectrum at $O(1 / J)$ :

$$
\begin{aligned}
\delta E_{s l(2)_{\gamma}}\left(\left\{n_{j}\right\},\left\{N_{j}\right\}, J\right)=\frac{1}{2 J}\left\{\sum_{j=1}^{M} N_{j}\left(N_{j}-1\right) \frac{\left(\tilde{\gamma}-n_{j} \sqrt{\lambda^{\prime}}\right)^{2}}{\omega_{n_{j}}^{2} \lambda^{\prime}}\right. \\
+\sum_{\substack{j, k=1 \\
j \neq k}}^{M} \frac{N_{j} N_{k}}{\omega_{n_{j}} \omega_{n_{k}} \lambda^{\prime}}\left\{3 \tilde{\gamma}^{2}+\tilde{\gamma}^{4}-\left(n_{j}+n_{k}\right) \tilde{\gamma}^{3} \sqrt{\lambda^{\prime}}+n_{j} n_{k} \lambda^{\prime}\left(1-n_{j} n_{k} \lambda^{\prime}\right)\right. \\
\left.\left.+\left(n_{j}+n_{k}\right) \tilde{\gamma} \sqrt{\lambda^{\prime}}\left(n_{j} n_{k} \lambda^{\prime}-2\right)+\lambda^{\prime}\left(n_{k} n_{k} \lambda^{\prime}-\tilde{\gamma}^{2}\right) \omega_{n_{j}} \omega_{n_{k}}\right\}\right\} .
\end{aligned}
$$

\section{Bethe equations}

We would now like to determine whether we can algorithmically derive a set of discretized Bethe equations that encode the above energy spectra in the near-pp-wave limit of the string theory, following the procedures outlined in Refs. 23, 29, 35, 24. Generally speaking, this rests on the premise that the spectrum is in fact described by the diffractionless scattering of elementary excitations on the worldsheet. The excitation momenta should then obey a fundamental equation (see, e.g., Ref. 24)

$$
p_{k} J=2 \pi n_{k}+\sum_{j \neq k} \theta\left(p_{k}, p_{j}\right),
$$

so that the spectrum is encoded in a two-body factorized $S$ matrix $S\left(p_{k}, p_{j}\right)$ :

$$
\theta\left(p_{k}, p_{j}\right)=-i \log S\left(p_{k}, p_{j}\right) .
$$

This generally means that the theory also admits an infinite number of hidden local charges arising as linear combinations of local dispersion relations $q_{r}$ :

$$
Q_{r}=\sum_{k} q_{r}\left(p_{k}\right) .
$$

Adopting this language, we write the $O(1 / J)$ corrections to the string energy spectrum in the near-pp-wave region as

$$
\delta \Delta\left(n_{k}, n_{j}, \gamma\right)=\lambda^{\prime} \sum_{\substack{j, k=1 \\ j \neq k}}^{S} \frac{J}{2 \pi} \frac{n_{k}}{\sqrt{1+\lambda^{\prime} n_{k}^{2}}} \theta\left(2 \pi n_{k} / J, 2 \pi n_{j} / J\right) .
$$

One issue arises, however, when interpreting $\sigma(x)$ (introduced in Section 3) as a density function $\rho$ of Bethe roots for the string sigma model. The following integral appears with incorrect normalization:

$$
\int_{C} d x \sigma(x) \sim \Delta+S-J .
$$


One way to fix this is to apply a nonlinear redefinition of the spectral parameter $29 \mid 35$ $\varphi \equiv x+T / x$, such that $T \equiv \frac{\lambda^{\prime}}{16 \pi^{2}}$, and $\rho(\varphi)=\sigma(x)$. Under this change of variables, the thermodynamic Bethe ansatz becomes

$$
\begin{aligned}
& 2 f d \varphi^{\prime} \frac{\rho\left(\varphi^{\prime}\right)}{\varphi-\varphi^{\prime}}=2 \pi\left(n_{k}-\gamma J\right)-p(\varphi) \\
& +f d \varphi^{\prime} \rho\left(\varphi^{\prime}\right)\left\{\frac{2 T}{\sqrt{\varphi^{\prime 2}-4 T} \sqrt{\varphi^{2}-4 T}}\left(\frac{x}{T-x x^{\prime}}-\frac{x^{\prime}}{T-x x^{\prime}}\right)\right. \\
& \left.\quad+4 \pi \gamma J T\left(\frac{1}{x^{2}-T}-\frac{1}{x^{\prime 2}-T}\right)\right\} .
\end{aligned}
$$

At this point, following Ref. 23, we should be able to recast the expression on the right-hand side in terms of the dispersion relations

$$
q_{r}(\varphi)=\frac{1}{\sqrt{\varphi^{2}-4 T}}\left(\frac{1}{2} \varphi+\frac{1}{2} \sqrt{\varphi^{2}-4 T}\right)^{1-r} .
$$

The undeformed sectors are known to arise from the geometric sum

$$
-2 f d \varphi^{\prime} \rho\left(\varphi^{\prime}\right)\left\{\sum_{r=1}^{\infty} T^{r}\left(q_{r+1}\left(\varphi^{\prime}\right) q_{r}(\varphi)-q_{r}\left(\varphi^{\prime}\right) q_{r+1}(\varphi)\right)\right\}
$$

while the deformation terms come from the combination

$$
-4 \pi \gamma J T f d \varphi^{\prime} \rho\left(\varphi^{\prime}\right)\left(q_{2}(\varphi)-q_{2}\left(\varphi^{\prime}\right)\right) .
$$

We therefore obtain the following thermodynamic Bethe ansatz:

$$
\begin{aligned}
& 2 f d \varphi^{\prime} \frac{\rho\left(\varphi^{\prime}\right)}{\varphi-\varphi^{\prime}}=2 \pi\left(n_{k}-\gamma J\right)-p(\varphi) \\
& -2 f d \varphi^{\prime} \rho\left(\varphi^{\prime}\right)\left\{\sum _ { r = 1 } ^ { \infty } T ^ { r } \left(q_{r+1}\left(\varphi^{\prime}\right) q_{r}(\varphi)\right.\right. \\
& \left.\left.\quad-q_{r}\left(\varphi^{\prime}\right) q_{r+1}(\varphi)\right)+2 \pi \gamma J T\left(q_{2}(\varphi)-q_{2}\left(\varphi^{\prime}\right)\right)\right\} .
\end{aligned}
$$

The above result can be understood to arise from the thermodynamic limit of a discrete ansatz for the deformed $s u(2)_{\gamma}$ sector of the theory:

$$
e^{i\left(p_{k}-2 \pi \gamma\right) J}=\prod_{\substack{j=1 \\ j \neq k}}^{S} \frac{\varphi\left(p_{k}\right)-\varphi\left(p_{j}\right)-i}{\varphi\left(p_{k}\right)-\varphi\left(p_{j}\right)+i} e^{-2 \pi i \gamma g^{2}\left(q_{2}\left(p_{k}\right)-q_{2}\left(p_{j}\right)\right)} \prod_{r=1}^{\infty} e^{-2 i \theta_{r}\left(p_{k}, p_{j}\right)},
$$

where $\theta_{r}\left(p_{k}, p_{j}\right) \equiv\left(g^{2} / 2\right)^{r}\left(q_{r}\left(p_{k}\right) q_{r+1}\left(p_{j}\right)-q_{r+1}\left(p_{k}\right) q_{r}\left(p_{j}\right)\right)$. We therefore find the following $\gamma$-dependent deformation contribution to the worldsheet $S$ matrix in the

a These expressions can be further simplified by rewriting the momenta in terms of the two constrained variables $x^{ \pm}$introduced in Ref. 4 For the sake of exposition, however, we keep the notation originally used in Ref. 22 . 
strong-coupling limit:

$$
\theta\left(p_{k}, p_{j}, \gamma\right) \approx-\frac{2}{\varphi\left(p_{k}\right)-\varphi\left(p_{j}\right)}-2 \sum_{r=1}^{\infty} \theta_{r}\left(p_{k}, p_{j}\right)-2 \pi \gamma g^{2}\left(q_{2}\left(p_{k}\right)-q_{2}\left(p_{j}\right)\right) .
$$

It is straightforward to check that this discrete Bethe ansatz correctly reproduces the $O(1 / J)$ energy shift in the $\operatorname{sl}(2)_{\gamma}$ sector of the string theory in the near-ppwave limit. The corresponding modifications to the discrete Bethe equations in the deformed $s u(2)_{\gamma}$ subsector are comparatively simple: they amount to an overall $\gamma$-dependent shift in the mode indices (see Ref. 22 for further details).

\section{Conclusions}

The investigation summarized in this letter was intended to provide a number of consistency checks on the methodology proposed in the literature for deriving discrete Bethe equations encoding the energy spectra of certain sectors of type IIB superstring theory on $A d S_{5} \times S^{5}$. While promising results had been established (see, e.g., Refs. 23, 29, 35), there was certainly no guarantee at the time that this methodology would work in the more complicated case of string theory on a TsTdeformed $A d S_{5}$ subspace. It was therefore satisfying to see that one could indeed find Bethe equations that properly reproduced (in a highly nontrivial manner) the leading $1 / J$ corrections to the energy spectrum away from the pp-wave limit. (See also Ref. 36, 37 for similar studies.)

Since the publication of Ref. 22 (on which the seminar reviewed here was based), the study of the (undeformed) $S$ matrix describing the string and gauge theory has greatly improved. A recent proposal by Beisert, Eden and Staudacher ${ }^{7}$ passes many nontrivial tests, and stands as a strong candidate for the complete $S$ matrix of the theory. It would be interesting to consider in this larger context the types of deformations discussed in Ref. 22, A first step would be to compute the leading finite- $\lambda$ corrections to the strong coupling limit of the $S$ matrix in these deformed string theories.

\section{Acknowledgments}

The author would like to thank Tristan McLoughlin for collaboration on Ref. 22, The seminar talk summarized here was based on this article. I.S. is the Marvin L. Goldberger Member at the Institute for Advanced Study, and is supported additionally by U.S. National Science Foundation grant PHY-0503584.

\section{References}

1. D. M. Hofman and J. M. Maldacena, J. Phys. A 39, 13095 (2006) arXiv:hep-th/0604135.

2. J. Maldacena and I. Swanson, arXiv:hep-th/0612079

3. J. A. Minahan and K. Zarembo, JHEP 0303, 013 (2003) arXiv:hep-th/0212208. 
4. N. Beisert, arXiv:hep-th/0511082.

5. R. A. Janik, Phys. Rev. D 73, 086006 (2006) arXiv:hep-th/0603038.

6. N. Beisert, R. Hernandez and E. Lopez, JHEP 0611, 070 (2006) arXiv:hep-th/0609044.

7. N. Beisert, B. Eden and M. Staudacher, J. Stat. Mech. 0701, P021 (2007) arXiv:hep-th/0610251.

8. G. Arutyunov, S. Frolov and M. Staudacher, JHEP 0410, 016 (2004) arXiv:hep-th/0406256.

9. R. G. Leigh and M. J. Strassler, Nucl. Phys. B 447, 95 (1995) arXiv:hep-th/9503121.

10. O. Lunin and J. M. Maldacena, JHEP 0505, 033 (2005) arXiv:hep-th/0502086].

11. S. A. Frolov, R. Roiban and A. A. Tseytlin, JHEP 0507, 045 (2005) arXiv:hep-th/0503192.

12. J. Plefka, arXiv:hep-th/0507136

13. S. Frolov, JHEP 0505, 069 (2005) arXiv:hep-th/0503201.

14. L. F. Alday, G. Arutyunov and S. Frolov, JHEP 0606, 018 (2006) arXiv:hep-th/0512253.

15. L. Freyhult, C. Kristjansen and T. Mansson, JHEP 0512, 008 (2005) arXiv:hep-th/0510221.

16. H. Y. Chen and S. Prem Kumar, JHEP 0603, 051 (2006) arXiv:hep-th/0511164.

17. H. Y. Chen and K. Okamura, JHEP 0602, 054 (2006) arXiv:hep-th/0601109].

18. N. Beisert and R. Roiban, JHEP 0508, 039 (2005) arXiv:hep-th/0505187].

19. M. Spradlin, T. Takayanagi and A. Volovich, JHEP 0511, 039 (2005) arXiv:hep-th/0509036.

20. N. P. Bobev, H. Dimov and R. C. Rashkov, arXiv:hep-th/0506063.

21. S. Ryang, JHEP 0511, 006 (2005) arXiv:hep-th/0509195].

22. T. McLoughlin and I. Swanson, JHEP 0608, 084 (2006) arXiv:hep-th/0605018].

23. G. Arutyunov, S. Frolov and M. Staudacher, JHEP 0410, 016 (2004) arXiv:hep-th/0406256.

24. M. Staudacher, JHEP 0505, 054 (2005) arXiv:hep-th/0412188.

25. N. Beisert and M. Staudacher, Nucl. Phys. B 727, 1 (2005) [arXiv:hep-th/0504190].

26. S. A. Frolov, R. Roiban and A. A. Tseytlin, Nucl. Phys. B 731, 1 (2005) arXiv:hep-th/0507021.

27. D. Berenstein, J. M. Maldacena and H. Nastase, JHEP 0204, 013 (2002) arXiv:hep-th/0202021.

28. G. Arutyunov and S. Frolov, JHEP 0502, 059 (2005) arXiv:hep-th/0411089.

29. V. A. Kazakov, A. Marshakov, J. A. Minahan and K. Zarembo, JHEP 0405, 024 (2004) arXiv:hep-th/0402207.

30. C. G. Callan, Jr., H. K. Lee, T. McLoughlin, J. H. Schwarz, I. J. Swanson and X. Wu, Nucl. Phys. B 673, 3 (2003) arXiv:hep-th/0307032.

31. C. G. Callan, Jr., T. McLoughlin and I. J. Swanson, Nucl. Phys. B 694, 115 (2004) arXiv:hep-th/0404007.

32. C. G. Callan, Jr., T. McLoughlin and I. J. Swanson, Nucl. Phys. B 700, 271 (2004) arXiv:hep-th/0405153.

33. I. J. Swanson, arXiv:hep-th/0505028

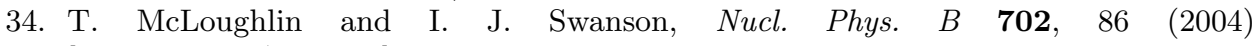
arXiv:hep-th/0407240.

35. N. Beisert, V. Dippel and M. Staudacher, JHEP 0407, 075 (2004) arXiv:hep-th/0405001.

36. I. J. Swanson, Nucl. Phys. B 709, 443 (2005) arXiv:hep-th/0410282.

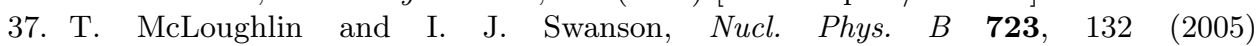


July 18, $2021 \quad$ 12:25 WSPC/INSTRUCTION FILE MPLA'Ian`Swanson

16 Ian Swanson

arXiv:hep-th/0504203. 